treatment, suggesting oxidative stress-induced di-sulfide bonds are also impaired in these mutants. We conclude that the oxidation of B2 box in PML plays a key role in PML-NBs assembly and sumoylation. We also identified a specific C-terminal cysteine in PML that participates in the formation of intermolecular disulfide bonds, yet not essential for PML-NBs assembly. This oxidized PML might contribute to maintain a specific redox environment in cells.

doi: http://dx.doi.org/10.7124/bc.000A04

\section{Z-1. Lysine methyltransferase SETDB1 and regulation of the three-dimensional organization of the genome during lung cancer progression}

V. Zakharova, G. Tellier, L. Del Maestro, V. Joliot and S. Ait-Si-Ali.

CNRS UMR7216 "Epigénétique et Destin Cellulaire" Université Paris Diderot Paris 7

zakharova.vlada@gmail.com

SETDB1 histone H3 lysine 9 (H3K9) methyltransferase (KMT) is known to be involved in tumorigenesis; however, the exact mechanisms of this involvement remain mainly unknown. SETDB1 regulates chromosomal conformation (Jiang et al., 2017); we recently found that SETDB1 interacts and could methylate members of the Cohesin complex. CTCF and Cohesin complex delimit Topologically Associated Domains (TADs) through direct binding at TAD boundaries. CTCF binding to chromatin is inhibited by $\mathrm{H} 3 \mathrm{~K} 9$ - and DNAmethylation. H3K9 trimethylation (H3K9me3) is a marker of metastasis in lung cancer patients, where SETDB1 gene is amplified. SETDB1 overexpression was associated with elevated cell growth and invasiveness of lung cancer cells (Rodriguez-Paredes et al., 2014). On the other hand, SETDB1 could act as a metastasis suppressor strongly downregulated in highly metastatic lung cancer cells. Aim: Here, we address the role of SETDB1 in the regulation of $3 \mathrm{D}$ genome architecture and gene expression patterns in an epithelial lung cancer cell line with different expression levels of SETDB1 (normal, low or high). Results: We found that SETDB1 regulates the epithelialmesenchymal transition that is a crucial in cancer progression and metastasis. We plan to perform CTCF, SETDB1 and H3K9me3 ChIPSeq assays combined with Bis-Seq to study DNA methylation at the CTCF binding sites in our cellular models. We will next correlate Hi-C maps with the defined epigenetic landscape. Conclusions: We expect that SETDB1 interacts with the Cohesin complex and affects CTCF occupancy at TAD boundaries, impacting 3D genome architecture and gene expression during during cancer progression.

doi: http://dx.doi.org/10.7124/bc.000A05

\section{Z-2. Investigating the nucleolar epigenetic code at ultrastructural level}

$\underline{\text { L. Zannino }}^{1}$, V. Bertone ${ }^{2}$, S. Siciliani ${ }^{1}$,

L. Saia ${ }^{1}$, M. Biggiogera ${ }^{1}$

${ }^{1}$ Laboratory of Cell Biology and Neurobiology, Department of Biology and Biotechnology, University of Pavia, Italy; ${ }^{2}$ Laboratory of Comparative 